\title{
Hemodynamics of Cerebral Aneurysms: Computational Analyses of Aneurysm Progress and Treatment
}

\author{
Woowon Jeong and Kyehan Rhee \\ Department of Mechanical Engineering, Myongji University, 38-2 Nam-Dong, Yongin-Si, Kyunggi-Do 449-728, Republic of Korea \\ Correspondence should be addressed to Kyehan Rhee, khanrhee@mju.ac.kr
}

Received 22 September 2011; Accepted 10 November 2011

Academic Editor: Eun Bo Shim

Copyright ( $) 2012$ W. Jeong and K. Rhee. This is an open access article distributed under the Creative Commons Attribution License, which permits unrestricted use, distribution, and reproduction in any medium, provided the original work is properly cited.

\begin{abstract}
The progression of a cerebral aneurysm involves degenerative arterial wall remodeling. Various hemodynamic parameters are suspected to be major mechanical factors related to the genesis and progression of vascular diseases. Flow alterations caused by the insertion of coils and stents for interventional aneurysm treatment may affect the aneurysm embolization process. Therefore, knowledge of hemodynamic parameters may provide physicians with an advanced understanding of aneurysm progression and rupture, as well as the effectiveness of endovascular treatments. Progress in medical imaging and information technology has enabled the prediction of flow fields in the patient-specific blood vessels using computational analysis. In this paper, recent computational hemodynamic studies on cerebral aneurysm initiation, progress, and rupture are reviewed. State-of-the-art computational aneurysmal flow analyses after coiling and stenting are also summarized. We expect the computational analysis of hemodynamics in cerebral aneurysms to provide valuable information for planning and follow-up decisions for treatment.
\end{abstract}

\section{Introduction}

Aneurysm is a vascular disease characterized by local dilatation of arterial walls. Aneurysms are frequently observed in the intracranial space and exhibit fusiform or saccular shapes. Some of aneurysms may grow, and rupture of cerebral aneurysms causes intracranial hemorrhage, which is associated with high mortality and morbidity [1-3]. In order to prevent the rupture of aneurysms, interventional thromboembolization treatment via the endovascular insertion of coils and stents may be applied as a prophylactic treatment. The recent development of neuroradiological and diagnostic imaging techniques has enabled aneurysms to be detected more frequently. Since much less than $1 \%$ of cerebral aneurysms rupture on an annual basis $[4,5]$, the demand for accurate prediction of aneurysm growth and rupture is increasing in order to select appropriate and immediate endovascular treatment.

The initiation, progression, and rupturing of aneurysms are related to the arterial wall remodeling: it is believed that they are all related to the complex interactions between biochemical and biomechanical factors. Pathological vessel wall remodeling involves various enzymes and proteins related to degeneration, inflammation, and repair: their expressions in arterial walls can be affected by hemodynamics. Blood flow imposes mechanical stress on the vessel wall, which may stimulate the functions of endothelial cells, affect the structural integrity of the endothelium, and affect the transport of various cells and enzymes in the blood stream to the endothelium. Therefore, hemodynamic forces and flow characteristics, such as recirculation [6], secondary flow [7], and jet impingement [8], are considered to be major mechanical factors related to the genesis and progression of vascular diseases. Among the hemodynamic parameters, wall shear stress (WSS) has been studied extensively, since endothelial cells actively sense and respond to WSS. In their recent review, Nixon et al. [9] clearly summarized the role of WSS in cerebral aneurysms and atherosclerosis.

Recent progress in medical imaging technology and improvements in computer equipment have enabled computational fluid dynamic (CFD) analysis to predict the hemodynamics of aneurysms with increased accuracy and reliability. Angiography image data can be converted to the three-dimensional (3D) vessel geometric data for computer 


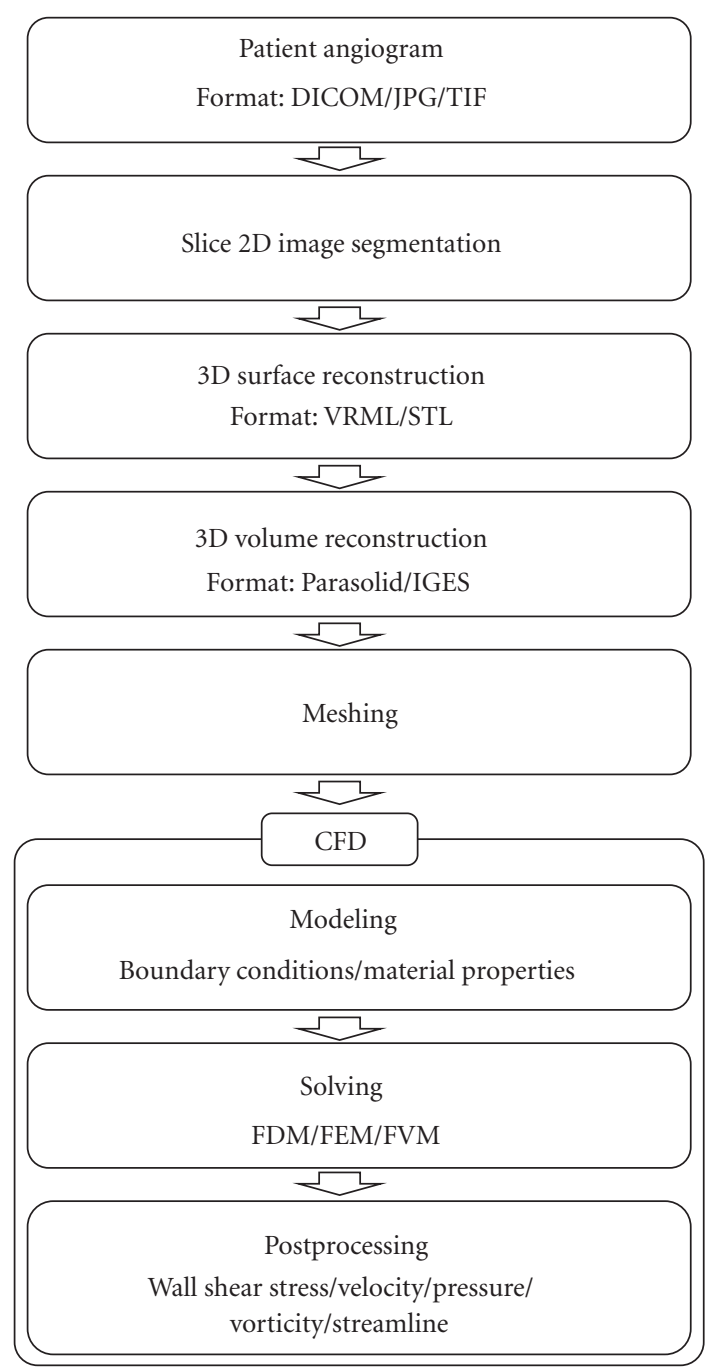

FIGURE 1: Flowchart of the computational hemodynamic analysis procedure based on patient-specific angiogram.

simulation; therefore, CFD analysis based on real aneurysm geometry has been progressed in recent years [10-14]. The basic process of computational hemodynamic analysis using a patient angiogram is illustrated in Figure 1. The sliced cross-sectional lumen images of a patient's vasculature are obtained using various imaging modalities, such as magnetic resonance angiography (MRA), computed tomography angiography (CTA), and 3D rotational angiography. The lumen of each cross-section image can be segmented and the luminal surface is reconstructed using splines, contour tilting, or other interpolation methods. Two-dimensional segmentation is inaccurate when the vessel axis is not perpendicular to the cross-sectional surface. Furthermore, manual automatic segmentation is operator dependent while automatic segmentation using threshold may yield topological defects and inaccuracies for the image with inhomogeneous image intensities. The state-of-the-art models for unsupervised full three-dimensional segmentation have been developed [15]. A region growing segmentation with automatic thresholding [16] and a component-based approach using a deformable model [17] are described in Cebral et al. [15] in detail. Based on the reconstructed surface contour, 3D solidvolume models are constructed using commercial 3D computer-aided design (CAD) programs. The reconstructed 3D volume models are divided into the computational grids by a meshing program. Some commercial software for preprocessing is outlined in Table 1 . The governing equations of a flow fields are solved using various numerical schemes, such as the finite differential method (FDM), finite element method (FEM), finite volume method (FVM), and lattice Boltzmann method (LBM). Various commercial CFD software programs including Fluent and Ansys CFX (ANSYS, Inc., Canonsburg, PA, USA), ADINA (ADINA R\&D, Inc., Watertown, MA, USA), COMSOL (COMSOL, Inc., Burlington, MA, USA), CFD-ACE (ESI Group, Paris, France), Flow3D (Flow Science, Inc., Pasadena, CA, USA), and STAR-CD (CD-adapco, Melville, NY, USA), are available. Calculated flow field variables can be rearranged in order to show various flow characteristics of interest using postprocessing procedures.

Patient-specific CFD analysis involves imaging data process, mesh construction, computational calculations, and postprocessing. In order to avoid difficulties associated with manual and time-consuming works of using commercial CFD packages, efforts of developing in-house CFD codes have been made. The whole pipeline from medical images to flow calculations has been developed in order to eliminate manual intervention and editing of data processing. Cebral et al. $[10,15,18,19]$ developed the pipeline for simulation based hemodynamics. It consists of vessel reconstruction, unstructured grid generation, numerical solver, and postprocessing. Proper boundary conditions and material properties are imposed on the blood vessel models. Patientspecific inflow data provide important information which determines the accuracy of CFD calculations. In vivo patientspecific inflow boundary conditions can either be obtained through invasive measurement using a catheter or medical imaging. Karmonik et al. [20-22] measured blood flow waveform in cerebral arteries by phase contrast MRI, and provided the measured blood flow profile at the inlet boundary conditions for CFD simulation. They successfully simulated blood flow in cerebral arteries using 3D digital subtraction angiography and phase contrast MRI. Specifying outflow boundary condition also requires careful attentions, and the appropriate impedances of the distal vasculature for multiple outlets should be provided.

If the hemodynamic factors affecting aneurysm etiology are elucidated, CFD analysis based on the patient-specific images data will provide a better understanding and diagnosis of aneurysm progress and rupture. The performance of endovascular stents and coils can be evaluated by analyzing aneurysmal flow alteration as a result of interventional treatment. Furthermore, prediction of blood flow using CFD analysis can be used to plan interventional therapies. In this paper, we review recent computational hemodynamic studies on cerebral aneurysm initiation, growth, and rupture, as well as computational studies on aneurysmal flow alterations induced by interventional treatment with coils and stents. 
TABLE 1: Some commercial preprocessing software for patient-specific CFD analysis and its role.

\begin{tabular}{lcccc}
\hline Software & $\begin{array}{c}\text { Slice 2D image } \\
\text { segmentation }\end{array}$ & $\begin{array}{c}\text { 3D surface } \\
\text { reconstruction }\end{array}$ & $\begin{array}{c}\text { 3D volume } \\
\text { reconstruction }\end{array}$ & Meshing \\
\hline 3DMax (Autodesk Inc., San Rafael, CA) & Yes & Yes & Yes & No \\
3D-Doctor (Able Software Corp., Lexington, MA) & Yes & Yes & Yes & No \\
Mimics (Materialise Group, Leuven, Belgium) & Yes & Yes & Yes & No \\
Insight Toolkit (Kitware Inc., Clifton Park, NY) & Yes & Yes & Yes & No \\
3D Slicer (MIT, Boston, MA) & Yes & Yes & Yes & No \\
SolidWorks (Dassault Systems, Concord, MA) & No & No & Yes & No \\
Pro-Engineer (PTC, Needham, MA) & No & No & Yes & No \\
CATIA (Dassault Systems, Velizy-Villacoublay, France) & No & No & Yes & No \\
3-matic (Materialise Group, Leuven, Belgium) & No & No & Yes & Yes \\
Hypermesh (Altair Engineering Inc., Troy, MI) & No & No & Yes & Yes \\
Gambit (ANSYS Inc., Canonsburg, PA) & No & No & Yes & Yes \\
ICEM-CFD (ANSYS Inc., Canonsburg, PA) & No & No & Yes & Yes \\
Gridgen (Pointwise Inc., Fort Worth, TX) & No & No & Yes & Yes \\
\hline
\end{tabular}

\section{Aneurysm Initiation}

Most cerebral aneurysms are observed at arterial bifurcations and branches or at the outer walls of arterial curvatures $[23,24]$. The localized occurrence of cerebral aneurysms prompted the hemodynamic research on aneurysm formation. Since CFD analysis provides detailed hemodynamic information at bifurcations $[25,26]$ and the outer walls of curved arteries $[27,28]$, many studies have attempted to identify an appropriate hemodynamic parameter correlated with pathological aneurysm formation.

Flow impingement on the apex of bifurcations and sharply curved vessels generates unstable helical flow patterns near the impinged wall. Repetitive flow impingement against the vessel wall under pulsatile flows may induce fatigue, potentially causing morphological and functional changes in the endothelium may occur in this region. The distributions of pressure and shear stress near the impingement point are shown in Figure 2. The local pressure increase at the impingement point is caused by the conversion of fluid kinetic energy to static wall pressure. Previous studies reported that the local pressure increases at arterial bifurcations and bends are less than $1-2 \%$ of intravascular pressure $[29,30]$. However, high spatial pressure gradients may affect endothelial remodeling.

WSS near the impingement point is high (hundreds of dyne $/ \mathrm{cm}^{2}[31]$ ), and its spatial gradient is also very large. Previous studies using animal experiments have demonstrated that high WSS contributes to the genesis of cerebral aneurysms via degenerative changes in endothelium $[32,33]$. Other studies have also shown that elevated WSS affects to the various degenerative changes of vessel walls [34-40].

Meng et al. [26] surgically created carotid bifurcations in a dog animal model and found that the spatial histological features of walls are correlated with hemodynamic variables calculated by CFD analysis. Their results show that the localization of destructive wall remodeling, which resembles the initiation of aneurysms, is correlated with a combination

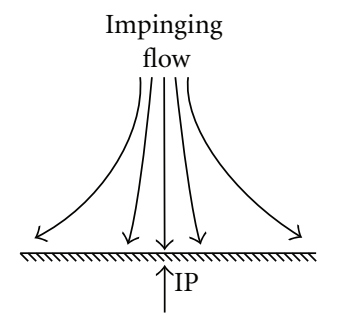

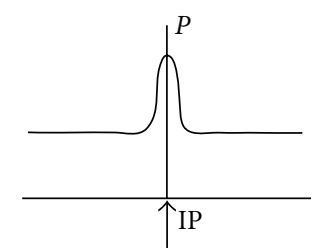

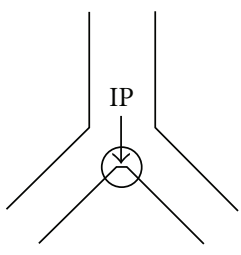

Bifurcation

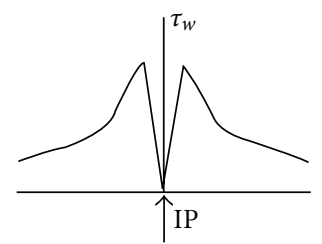

FIgURE 2: Schematic of pressure $(P)$ and WSS $\left(\tau_{w}\right)$ distribution near the jet impingement point (IP) of curved and bifurcated blood vessels.

of high WSS and a high WSS gradient. Mantha et al. [37] constructed computational models of carotid artery lateral aneurysms based on the patient 3D angiography data, and performed CFD analysis was performed for the parent vessel prior to aneurysm formation. They found significant correlations between the temporal directional changes of WSS and the location of aneurysm formation.

Since the endothelium senses WSS and actively responds to mechanical stress [34-36, 38, 40], vascular remodeling that initiates aneurysm formation may be related to high WSS magnitude or high spatial and temporal variations in WSS. Although it is difficult to pinpoint the hemodynamic 
variables responsible for aneurysm initiation, complex flow fields near the flow impingement point, where high WSS magnitude, high temporal and spatial WSS gradients, and high pressure gradients are probably part of the prerequisite hemodynamic environment related to the initiation of cerebral aneurysms.

\section{Aneurysm Growth}

Histological studies have revealed degeneration of the intima and thinning of the media in the aneurysm vessel wall $[39,41]$. Furthermore, various proteolytic enzyme secretions such as elastase [42] and matrix metalloproteinase [43] contribute to degenerative wall remodeling. Pathological wall remodeling processes, which are related to the secretion of various enzymes, along with the inflammatory response of endothelium [44] and apoptosis of smooth muscle cells [45, 46], may be affected by hemodynamics [47]. CFD studies have been performed to investigate the hemodynamic factors affecting aneurysm growth. Many CFD studies have been performed on the growth and rupture of aneurysms using not only the ideal curved and bifurcated blood vessel models [31, 48-51], but also aneurysm models based on real patient data obtained by MRI or CT imaging [13, 14, 52, 53].

Feng et al. [54, 55] simulated the deformation and growth of an aneurysm bleb of a curved vessel in a simplified cerebral artery model. They assumed that aneurysm growth was related to degeneration of the arterial wall caused by high WSS. Aneurysm formation was modeled as vessel wall deformation due to reduced wall stiffness using fluid solid interaction (FSI). The WSS distributions were calculated, and the Young's modulus of the arterial wall was assumed to be reduced if the WSS was larger than the threshold value. The drawback of their methodology is that the mechanical properties of an arterial wall could not be modeled as a simple function of WSS. Furthermore, arterial remodeling could not be simply modeled by using a vessel wall expansion model based on FSI, and the temporal progression of aneurysm growth was not considered. Boussel et al. [56] calculated the WSS of the intracranial aneurysm on the basis of the patient's MR angiography data, and correlated WSS with aneurysm growth by using MR images at 2 different time points (mean $16.4 \pm 7.4$ months between the 2 time points): they found a significant correlation between low time-averaged WSS and aneurysm growth.

Atherosclerotic vascular wall changes due to low and oscillatory WSS have been studied extensively, and the role of hemodynamics on atherogenesis is well established [5759]. Although vessel wall remodeling in aneurysm growth is different from atherosclerotic wall remodeling [46, 60], inflammatory responses due to disturbed flow or decreased WSS may affect the aneurysm growth and rupture via degenerative vascular wall remodeling. Low WSS $\left(<4 \mathrm{dyne} / \mathrm{cm}^{2}\right)$ causes endothelial proliferation [61] and apoptosis [62]. Moreover, low WSS induced by slow flow recirculation in the complex flow fields in the aneurysmal bifurcations $[53,63]$ is positively correlated with aneurysm growth. One study found that the WSS of the aneurysms is significantly lower than that of the surrounding vasculature [53]. Furthermore, a relationship between local aneurysm growth and areas of low WSS at the intraluminal surface was found using patient aneurysm models $[53,56]$.

The complex flow patterns accompanying low and oscillating WSS may be correlated with aneurysm growth. Slight out-pouching of the aneurysm wall may induce a disturbed flow region, and aneurysm growth can be accelerated due to low and oscillatory WSS in the expanded blood vessel via a positive feedback mechanism. Vascular wall remodeling maintaining the WSS at a constant level favors the high WSS hypothesis of aneurysm growth. However, it cannot explain aneurysm growth, because intracranial arteries have different vessel wall structures from the arteries in which arterial remodeling is observed [64-67].

\section{Aneurysm Rupture}

Aneurysm rupture occurs when wall tension exceeds the mechanical strength of wall tissue. Wall tension is proportional to the intramural pressure and radius, and inversely proportional to the walls thickness of a spherical balloon. Therefore, high pressure, large aneurysm size, and thin wall increase wall tension. Local weakening of the aneurysm wall, which is characterized by thinning of the media and a lack of collagen fibers, is closely related to the pathological wall remodeling. Furthermore, the hemodynamic environment may affect the wall remodeling process. Therefore, hemodynamic forces, including high pressure and WSS, may directly influence wall breakage, while low WSS and disturbed flow patterns may affect the aneurysm wall weakening via wall remodeling over an extended period.

Strong flow impingement is suspected as a hemodynamic factor responsible for aneurysm rupture. Cebral et al. [52] performed a CFD study using 62 patient-specific models. They found out that flow patterns are more complex and the impingement jet is narrower in ruptured aneurysms compared to unruptured aneurysms. Recently, the same group extended the previous study by including 210 intracranial aneurysms in 128 consecutive patients and more hemodynamic characteristics were investigated [68]. A quantitative hemodynamic study showed that concentrated inflow jets, small impingement regions, complex flow patterns, and unstable flow patterns were correlated with a clinical history of prior aneurysm rupture. Therefore, strong inflow jets from the parent vessel to the aneurysm sac might provide a hemodynamic environment prone to aneurysm rupture. Blood impingement on the wall generates impact force and high WSS, which could affect the fatigue of the vessel wall. The site of flow impact includes locally elevated pressure and a high WSS gradient. The contribution of pressure elevation due to the impinging flow jet is small $[31,69]$, but the effect of impact force remains unknown. Shojima et al. [53] calculated the WSS in the human middle cerebral artery using CFD analysis. They reported that the spatially averaged WSS of the aneurysm region at the peak systole is significantly higher (approximately 2 times higher) in ruptured models than that in unruptured models. High spatially averaged WSS in the ruptured cases is due to the high WSS at the body or the neck of aneurysm caused by direct flow 
stream from a parent artery. Therefore, high WSS may not be correlated with aneurysm rupture. The WSS at the tip of the aneurysm, where it is more vulnerable to rupture, is lower in the ruptured cases. But their computational results may not detect secondary flows and other detailed features of the intraaneurysmal flow pattern because the computational vessel models reconstructed in their study are extremely truncated. Castro et al. [18] studied the influence of the upstream parent artery geometry on intraaneurysmal hemodynamics. They showed that the reconstructed models using the truncated parent vessel underestimated the WSS and shifted the impact zone to the neck. Therefore, further studies should be required to clarify the effects of low and high WSS on aneurysm rupture.

Complex unstable flow accompanying low and oscillatory flow may be responsible for vessel wall remodeling associated with rupture [70], since it is correlated with apoptotic wall remodeling [62]. Valencia et al. [71] showed that low WSS regions are larger in ruptured aneurysms than in unruptured ones. They also report a linear correlation between the average WSS on the aneurysm sac at peak systole with the area index, which was defined as the ratio between the aneurysm area and the artery areas. Lu et al. [72] performed CFD analysis using 3D reconstructed angiograms of both ruptured and unruptured cerebral aneurysms. In the ruptured group, the proportion of low WSS areas $(<1.5 \mathrm{~Pa})$ to the whole area of the aneurysm was significantly lower, while the oscillatory shear index was significantly higher. Therefore, large spatial and temporal variations in WSS within the complex unstable flow in the aneurysm sac might be related to aneurysm rupture.

Pressure may be a hemodynamic parameter influencing aneurysm rupture. Previous studies demonstrated that the complex flow pattern of the flow impingement around the aneurysm results in elevated pressure at the aneurysm [30, $48,69]$, although it is very low compared to systemic pressure. A large pressure gradient along a wall near the impingement point may affect endothelial functioning and remodeling. Torii et al. [73, 74] studied the effects of high blood pressure on WSS in aneurysm models using image-based FSI modeling. Their results show that hypertensive blood pressure causes significant changes in WSS distribution on the aneurysm wall. Thus, hypertension may affect aneurysm wall damage.

Since aneurysm rupture is not correlated with the abnormally high local stress, a loss of wall integrity due to vascular wall remodeling and increased wall tension due to systemic pressure may be responsible for aneurysm rupture. Isaksen et al. [75] calculated wall tension and displacement using FSI for the elastic cerebral aneurysm wall. They show that the areas of maximum wall tension and displacement are located where aneurysms are most vulnerable to rupture. Local changes of wall thickness and mechanical property caused by aneurysm wall remodeling were not considered since they assumed a uniform aneurysm wall thickness. Local wall thickness and the properties of the aneurysm wall are hard to measure, which limits the estimation of wall tension.

\section{Aneurysm Coiling}

Until the early 1990s, surgical clipping was used to obliterate the aneurysm sac. However, endovascular treatments such as coil embolization and stenting have been used since the development of nonsurgical endovascular approaches. Coils packed into the aneurysm sac induce flow stasis and thrombus formation [76-78], and scar tissue forms as a result of the foreign body reaction completing the aneurysmal occlusion $[76,79,80]$. It is difficult to completely fill an aneurysmal cavity due to the complex shape of the aneurysm sac and coil compaction due to hemodynamic force. Incomplete embolization induces residual flow from the parent vessel and contributes to the recurrence of the aneurysm. Accordingly, hemodynamics after coil embolization is a highly relevant factor for predicting the recurrence and regrowth of aneurysms.

Byun and Rhee [81] studied hemodynamic changes due to partially blocked lateral aneurysms, and investigated the effects of aneurysm shape and parent vessel geometry using CFD analysis. A computer simulation of a terminal aneurysm was also performed, and the effects of coil density were explored [76]. Since aneurysm wall remodeling and flow stasis may be important, aneurysmal WSS was investigated taking fluid (blood) and solid (vessel wall) interactions into consideration [82]. In these analyses, coils were modeled as a solid material (block element); these analyses might be valid for completely thromboembolized coils.

In order to investigate the flow inside the coil, the coil was modeled as a porous medium. CFD analysis was performed using aneurysm images of the real patient, and the influence of multiple coils on intra-aneurysmal hemodynamics was investigated $[83,84]$. Porous medium modeling might be useful in the early stage of coil insertion, but the thrombus formation inside of a coil bundle should be modeled during the embolization process after endovascular treatment. Computational simulation of the blood clotting inside the idealized lateral aneurysm model was performed on the basis of the viscosity model defined as a function of both residence time and clotting fluid concentration [85]. However, further refinement of the clotting model is required, since its validation is incomplete.

The interaction between hemodynamics and the coil is the major physical cause of coil compaction and dislocation, which induce the recanalization and growth of the aneurysm. The interaction between coils and flow has been simulated. One application of these simulations is to simulate coil deployment during the initial stage of coil intervention. The results from such simulations may help provide patientspecific guidelines for coil selection and predict postoperative prognosis. Recently, Wei et al. [86] simulated coil deployment and deformation while considering reactive fluid force, and computed flow fields for coil filled and coil free domains. Since simulations of aneurysmal hemodynamics in the interaction between coils and flow are limited, further studies should be performed to elucidate the role of hemodynamics on coil compaction and dislocation. 


\section{Aneurysm Stenting}

Stents have been used as scaffolds to keep coils in the cavities of fusiform and wide-neck aneurysms. At present, it is believed that stents alone can be used to reduce the flow from the parent artery and to thromboembolize the aneurysm sac. Although thromboembolization with stents may be less effective than that with coils, the advantages of stenting include the ability to stabilize the aneurysm without touching the aneurysm sac and to block the aneurysm neck by inducing neointima formation over the stent surface. Predicting the hemodynamic changes after stenting could be helpful for designing and selecting stents effective for aneurysm embolization.

Flow patterns, vorticity, slip velocity, and WSS are relevant hemodynamic parameters affecting the thromboembolization efficiency of stents. Flow visualization methods have been used in aneurysm phantom models to elucidate the effects of stent porosity [87-90]. Quantitative experimental methods using particle image velocimetry $[91,92]$ and laser Doppler velocimetry $[93,94]$ have also been used to evaluate alterations in hemodynamics according to different vessel geometries and stent designs.

Computational analyses have been performed to elucidate the flow field alterations caused by stents and to evaluate their ability to divert flow and induce stasis inside of aneurysmal cavities $[95,96]$. Patient-specific aneurysm models have also been analyzed to elucidate the flow alterations caused by stenting [11, 70, 97-102]. Recently, Cebral et al. [103] performed CFD analysis on the cerebral aneurysms which are apparently successfully treated using a stent but have been complicated by later aneurysm hemorrhage. They showed that flow diversion stent can cause intra-aneurysmal pressure increase, which can potentially lead to rupture. Among the challenges in the CFD simulation of stented aneurysms, there are major difficulties related to the meshing of stents due to the scale differences between the artery and stent strut thickness. Some adaptive embedding techniques have been developed to resolve such meshing difficulties [104-106]: vessel walls are treated with body-fitted unstructured grids, and stents are embedded in the grids; furthermore, adaptive meshing refinement is performed near the stents.

The flow of stented lateral and terminal aneurysms have been simulated by modeling a stent as a porous medium [107]. In this study, a rough model of stent geometry can reproduce flow features quantitatively as well as qualitatively. LBMs considering velocity reduction caused by stent implantation in advance using the stent positioning effect have also been developed to predict the thromboembolization of stented aneurysms [108-110]. The simulation of stent deployment is also challenging. In some models, an elastic cylindrical support surface is generated along the parent vessel that deforms until it comes into contact with the vessel wall $[104,111]$. The inlet and outlet boundary conditions, as well as the parent vessel wall elasticity before and after the stenting, should be specified accurately. Further studies are required to model stent deployment as well as vessel wall and flow field alterations due to stenting, neointima formation along the stent, and thromboembolization modeling.

\section{Discussion and Conclusions}

Experimental hemodynamic studies have been performed using various cerebral aneurysm models in order to investigate the role of fluid dynamics in the etiology of aneurysms $[48,94,111-115]$ and endovascular treatment $[31,105,116]$. These studies have attempted to find correlations between complex hemodynamic variables and cerebral aneurysm formation, growth and rupture with idealized model geometries. Hemodynamic alterations after stenting and coiling are also observed in in vitro models $[81,90]$ as well as in surgically created aneurysms in animal models [117]. Since the details of flow patterns are significantly affected by vessel and aneurysm geometries, realistic vascular geometry is believed to be one of the most important factors for precisely analyzing flow in aneurysms and arteries.

Recently, detailed patients' vessel geometries have become available as a result of the progress of high-resolution angiography techniques. Moreover, and the recent advances in information technology have enabled the prediction of hemodynamics in patient-specific blood vessels using CFD analysis. Difficulties in accurately determining blood vessel geometry from patient angiograms are related to the segmentation of the lumen, defining the calculation domain, and computational mesh formation. Since these difficulties require a great deal of manual, time-consuming, and operator-dependent work to be overcome, efforts for developing in-house codes for patient-specific CFD analyses have been made.

Other difficulties in CFD analysis are related to defining the material properties and boundary conditions for realistic in vivo arterial blood flow. The consideration of the rheological viscosity characteristics of blood and vessel wall elasticity in arterial blood flow analysis has remained controversial over the last few decades. Even though nonNewtonian viscosity characteristics are not important for analyzing blood flow in large arteries [11, 118-121], they may influence the WSS distributions in aneurysmal flow [10]. The implementation of non-Newtonian viscosity laws in CFD calculations requires relatively insignificant efforts and computational loads, although validating the viscosity law in complex flow fields may be difficult. Taking the wall elasticity of arteries into consideration in computational analysis requires additional information on vessel radial wall motion, local wall properties, and pressure waveforms - all of which are difficult to obtain. CFD analysis using dynamic angiography has been attempted [122-124] to define radial vessel wall motion. Specifying outflow boundary conditions also requires careful attentions, and the appropriate impedances of the distal vasculature for multiple outlets should be provided.

Even though accurate vessel geometry can be obtained using high-resolution medical imaging modalities and hemodynamic analysis can be performed with the help of state-of-the-art computing techniques, the application of hemodynamic parameters for predicting aneurysm growth and rupture is still limited. Although information regarding vessel wall structure is very useful, it is difficult to obtain in vivo information of aneurysm wall structures. A vascular 
wall-remodeling model based on cell mechanobiology has been developed on the basis of FSI [125-127]. In this particular growth and remodeling model, hemodynamic analysis provides the wall tractions for wall mechanics computation to refine wall properties and geometries. The solution iterated between fluid and solid wall mechanics, and stress mediated-growth and aneurysmal remodeling have been analyzed. Modeling the changes in properties, configurations, and mass fractions of the aneurysmal wall due to mechanical stress requires further study [49, 125, 128]. Moreover, difficulties related to adaptive mesh generation [129-131] and boundary conditions for describing wall traction should be considered when computationally analyzing vascular wall remodeling.

Patient-specific hemodynamic simulations would provide valuable information for planning and follow-up decisions in cerebral aneurysm treatment. Additional effort is required to develop fast and accurate computational methodologies using high-resolution medical images to apply CFD simulation in cerebral aneurysm diagnostic and intervention planning tools. In addition, fundamental mechanobiological studies on the effects of hemodynamics on aneurysm growth, rupture, and thromboembolization should be performed in order to refine and complete the computational modeling.

\section{Acknowledgment}

This paper was supported by a Grant from the Korean Health Technology R\&D Project, Ministry of Health and Welfare, Republic of Korea (A111101).

\section{References}

[1] M. Kaminogo, M. Yonekura, and S. Shibata, "Incidence and outcome of multiple intracranial aneurysms in a defined population," Stroke, vol. 34, no. 1, pp. 16-21, 2003.

[2] F. H. H. Linn, G. J. E. Rinkel, A. Algra, and J. Van Gijn, "Incidence of subarachnoid hemorrhage: role of region, year, and rate of computed tomography: a meta-analysis," Stroke, vol. 27, no. 4, pp. 625-629, 1996.

[3] H. R. Winn, J. A. Jane, J. Taylor, D. Kaiser, and A. G. W. Britz, "Prevalence of asymptomatic incidental aneurysms: review of 4568 arteriograms," Journal of Neurosurgery, vol. 96, no. 1, pp. 43-49, 2002.

[4] S. Juvela, "Natural history of unruptured intracranial aneurysms: risks for aneurysm formation, growth, and rupture," Acta Neurochirurgica, Supplement, no. 82, pp. 2730, 2002.

[5] D. O. Wiebers, "Unruptured intracranial aneurysms: natural history, clinical outcome, and risks of surgical and endovascular treatment," Lancet, vol. 362, no. 9378, pp. 103-110, 2003.

[6] J. Cao and S. E. Rittgers, "Particle motion within in vitro models of stenosed internal carotid and left anterior descending coronary arteries," Annals of Biomedical Engineering, vol. 26, no. 2, pp. 190-199, 1998.

[7] S. Z. Zhao, X. Y. Xu, A. D. Hughes et al., "Blood flow and vessel mechanics in a physiologically realistic model of a human carotid arterial bifurcation," Journal of Biomechanics, vol. 33, no. 8, pp. 975-984, 2000.
[8] A. P. Yoganathan, J. Ball, Y. R. Woo et al., "Steady flow velocity measurements in a pulmonary artery model with varying degrees of pulmonic stenosis," Journal of Biomechanics, vol. 19, no. 2, pp. 129-146, 1986.

[9] A. M. Nixon, M. Gunel, and B. E. Sumpio, "The critical role of hemodynamics in the development of cerebral vascular disease: a review," Journal of Neurosurgery, vol. 112, no. 6, pp. 1240-1253, 2010.

[10] J. R. Cebral, M. A. Castro, S. Appanaboyina, C. M. Putman, D. Millan, and A. F. Frangi, "Efficient pipeline for imagebased patient-specific analysis of cerebral aneurysm hemodynamics: technique and sensitivity," IEEE Transactions on Medical Imaging, vol. 24, no. 4, pp. 457-467, 2005.

[11] D. A. Steinman, J. S. Milner, C. J. Norley, S. P. Lownie, and D. W. Holdsworth, "Image-based computational simulation of flow dynamics in a giant intracranial aneurysm," American Journal of Neuroradiology, vol. 24, no. 4, pp. 559-566, 2003.

[12] C. A. Taylor and D. A. Steinman, "Image-based modeling of blood flow and vessel wall dynamics: applications, methods and future directions," Annals of Biomedical Engineering, vol. 38, no. 3, pp. 1188-1203, 2010.

[13] R. Torii, M. Oshima, T. Kobayashi, K. Takagi, and T. E. Tezduyar, "Fluid-structure interaction modeling of a patient-specific cerebral aneurysm: influence of structural modeling," Computational Mechanics, vol. 43, no. 1, pp. 151159, 2008.

[14] A. Valencia, D. Ledermann, R. Rivera, E. Bravo, and M. Galvez, "Blood flow dynamics and fluid-structure interaction in patient-specific bifurcating cerebral aneurysms," International Journal for Numerical Methods in Fluids, vol. 58, no. 10, pp. 1081-1100, 2008.

[15] J. R. Cebral, M. A. Castro, O. Soto, R. Löhner, and N. Alperin, "Blood-flow models of the circle of Willis from magnetic resonance data," Journal of Engineering Mathematics, vol. 47, no. 3-4, pp. 369-386, 2003.

[16] J. R. Cebral and R. Lhner, "From medical images to anatomically accurate finite element grids," International Journal for Numerical Methods in Engineering, vol. 51, no. 8, pp. 9851008, 2001.

[17] P. J. Yim, J. J. Cebral, R. Mullick, H. B. Marcos, and P. L. Choyke, "Vessel surface reconstruction with a tubular deformable model," IEEE Transactions on Medical Imaging, vol. 20, no. 12, pp. 1411-1421, 2001.

[18] M. A. Castro, C. M. Putman, and J. R. Cebral, "Computational fluid dynamics modeling of intracranial aneurysms: effects of parent artery segmentation on intra-aneurysmal hemodynamics," American Journal of Neuroradiology, vol. 27, no. 8, pp. 1703-1709, 2006.

[19] P. Yim, K. DeMarco, M. A. Castro, and J. Cebral, "Characterization of shear stress on the wall of the carotid artery using magnetic resonance imaging and computational fluid dynamics," Studies in Health Technology and Informatics, vol. 113, pp. 412-422, 2005.

[20] C. Karmonik, C. Yen, O. Diaz, R. Klucznik, R. G. Grossman, and G. Benndorf, "Temporal variations of wall shear stress parameters in intracranial aneurysms-importance of patientspecific inflow waveforms for CFD calculations," Acta Neurochirurgica, vol. 152, no. 8, pp. 1391-1398, 2010.

[21] C. Karmonik, C. Yen, R. G. Grossman, R. Klucznik, and G. Benndorf, "Intra-aneurysmal flow patterns and wall shear stresses calculated with computational flow dynamics in an anterior communicating artery aneurysm depend on knowledge of patient-specific inflow rates," Acta Neurochirurgica, vol. 151, no. 5, pp. 479-485, 2009. 
[22] M. Spiegel, T. Redel, Y. J. Zhang et al., “Tetrahedral vs. polyhedral mesh size evaluation on flow velocity and wall shear stress for cerebral hemodynamic simulation," Computer Methods in Biomechanics and Biomedical Engineering, vol. 14, no. 1, pp. 9-22, 2011.

[23] F. Bonneville, N. Sourour, and A. Biondi, "Intracranial Aneurysms: an overview," Neuroimaging Clinics of North America, vol. 16, no. 3, pp. 371-382, 2006.

[24] A. L. Rhoton, "Aneurysms," Neurosurgery, vol. 51, no. 4, pp. 121-158, 2002.

[25] L. Gao, Y. Hoi, D. D. Swartz, J. Kolega, A. Siddiqui, and H. Meng, "Nascent aneurysm formation at the basilar terminus induced by hemodynamics," Stroke, vol. 39, no. 7, pp. 2085 2090, 2008.

[26] H. Meng, Z. Wang, Y. Hoi et al., "Complex hemodynamics at the apex of an arterial bifurcation induces vascular remodeling resembling cerebral aneurysm initiation," Stroke, vol. 38, no. 6, pp. 1924-1931, 2007.

[27] T. A. Altes, H. J. Cloft, J. G. Short et al., "Creation of saccular aneurysms in the rabbit: a model suitable for testing endovascular devices," American Journal of Roentgenology, vol. 174, no. 2, pp. 349-354, 2000.

[28] N. H. Fujiwara, H. J. Cloft, W. F. Marx, J. G. Short, M. E. Jensen, and D. F. Kallmes, "Serial angiography in an elastaseinduced aneurysm model in rabbits: evidence for progressive aneurysm enlargement after creation," American Journal of Neuroradiology, vol. 22, no. 4, pp. 698-703, 2001.

[29] H. Meng, E. Metaxa, Z. Wang et al., "Vascular response to impinging blood flow," in Proceedings of the BMES Annual Fall Meeting, Baltimore, MD, USA, 2005.

[30] M. Shojima, M. Oshima, K. Takagi et al., "Role of the bloodstream impacting force and the local pressure elevation in the rupture of cerebral aneurysms," Stroke, vol. 36, no. 9, pp. 1933-1938, 2005.

[31] Y. Hoi, H. Meng, S. H. Woodward et al., "Effects of arterial geometry on aneurysm growth: three-dimensional computational fluid dynamics study," Journal of Neurosurgery, vol. 101, no. 4, pp. 676-681, 2004.

[32] S. Fukuda, N. Hashimoto, H. Naritomi et al., "Prevention of rat cerebral aneurysm formation by inhibition of nitric oxide synthase," Circulation, vol. 101, no. 21, pp. 2532-2538, 2000.

[33] S. Kondo, N. Hashimoto, H. Kikuchi, F. Hazama, I. Nagata, and H. Kataoka, "Cerebral aneurysms arising at nonbranching sites: an experimental study," Stroke, vol. 28, no. 2, pp. 398-404, 1997.

[34] S. Chien, S. Li, and J. Y. J. Shyy, "Effects of mechanical forces on signal transduction and gene expression in endothelial cells," Hypertension, vol. 31, no. 1, pp. 162-169, 1998.

[35] P. F. Davies, "Flow-mediated endothelial mechanotransduction,” Physiological Reviews, vol. 75, no. 3, pp. 519-560, 1995.

[36] P. F. Davies, K. A. Barbee, M. V. Volin et al., "Spatial relationships in early signaling events of flow-mediated endothelial mechanotransduction," Annual Review of Physiology, vol. 59, pp. 527-549, 1997.

[37] A. Mantha, C. Karmonik, G. Benndorf, C. Strother, and R. Metcalfe, "Hemodynamics in a cerebral artery before and after the formation of an aneurysm," American Journal of Neuroradiology, vol. 27, no. 5, pp. 1113-1118, 2006.

[38] J. Y. J. Shyy, "Mechanotransduction in endothelial responses to shear stress: review of work in Dr. Chien's laboratory," Biorheology, vol. 38, no. 2-3, pp. 109-117, 2001.

[39] W. E. Stehbens, "Histopathology of cerebral aneurysms," Archives of neurology, vol. 8, pp. 272-285, 1963.
[40] O. Traub and B. C. Berk, "Laminar shear stress: mechanisms by which endothelial cells transduce an atheroprotective force," Arteriosclerosis, Thrombosis, and Vascular Biology, vol. 18, no. 5, pp. 677-685, 1998.

[41] W. E. Stehbens, "Etiology of intracranial berry aneurysms," Journal of Neurosurgery, vol. 70, no. 6, pp. 823-831, 1989.

[42] D. Chyatte and I. Lewis, "Gelatinase activity and the occurrence of cerebral aneurysms," Stroke, vol. 28, no. 4, pp. 799804, 1997.

[43] G. Bruno, R. Todor, I. Lewis, and D. Chyatte, "Vascular extracellular matrix remodeling in cerebral aneurysms," Journal of Neurosurgery, vol. 89, no. 3, pp. 431-440, 1998.

[44] H. D. Intengan and E. L. Schiffrin, "Vascular remodeling in hypertension: roles of apoptosis, inflammation, and fibrosis," Hypertension, vol. 38, no. 3, pp. 581-587, 2001.

[45] A. Hara, N. Yoshimi, and H. Mori, "Evidence for apoptosis in human intracranial aneurysms," Neurological Research, vol. 20, no. 2, pp. 127-130, 1998.

[46] S. Kondo, N. Hashimoto, H. Kikuchi, F. Hazama, I. Nagata, and H. Kataoka, "Apoptosis of medial smooth muscle cells in the development of saccular cerebral aneurysms in rats," Stroke, vol. 29, no. 1, pp. 181-189, 1998.

[47] B. L. Langille, "Arterial remodeling: relation to hemodynamics," Canadian Journal of Physiology and Pharmacology, vol. 74, no. 7, pp. 834-841, 1996.

[48] A. C. Burleson, C. M. Strother, V. T. Turitto, H. H. Batjer, S. Kobayashi, and R. E. Harbaugh, "Computer modeling of intracranial saccular and lateral aneurysms for the study of their hemodynamics," Neurosurgery, vol. 37, no. 4, pp. 774784, 1995.

[49] I. Chatziprodromou, A. Tricoli, D. Poulikakos, and Y. Ventikos, "Haemodynamics and wall remodelling of a growing cerebral aneurysm: a computational model," Journal of Biomechanics, vol. 40, no. 2, pp. 412-426, 2007.

[50] Y. Hoi, S. H. Woodward, M. Kim, D. B. Taulbee, and H. Meng, "Validation of CFD simulations of cerebral aneurysms with implication of geometric variations," Journal of Biomechanical Engineering, vol. 128, no. 6, pp. 844-851, 2006.

[51] B. Utter and J. S. Rossmann, "Numerical simulation of saccular aneurysm hemodynamics: influence of morphology on rupture risk," Journal of Biomechanics, vol. 40, no. 12, pp. 2716-2722, 2007.

[52] J. R. Cebral, M. A. Castro, J. E. Burgess, R. S. Pergolizzi, M. J. Sheridan, and C. M. Putman, "Characterization of cerebral aneurysms for assessing risk of rupture by using patientspecific computational hemodynamics models," American Journal of Neuroradiology, vol. 26, no. 10, pp. 2550-2559, 2005.

[53] M. Shojima, M. Oshima, K. Takagi et al., "Magnitude and role of wall shear stress on cerebral aneurysm: computational fluid dynamic study of 20 middle cerebral artery aneurysms," Stroke, vol. 35, no. 11, pp. 2500-2505, 2004.

[54] Y. Feng, S. Wada, K. I. Tsubota, and T. Yamaguchi, "The application of computer simulation in the genesis and development of intracranial aneurysms," Technology and Health Care, vol. 13, no. 4, pp. 281-291, 2005.

[55] Y. Feng, S. Wada, K. I. Tsubota, and T. Yamaguchi, "Growth of intracranial aneurysms arised from curved vessels under the influence of elevated wall shear stress-a computer simulation study," JSME International Journal, Series C, vol. 47, no. 4, pp. 1035-1042, 2004.

[56] L. Boussel, V. Rayz, C. McCulloch et al., "Aneurysm growth occurs at region of low wall shear stress: patient-specific 
correlation of hemodynamics and growth in a longitudinal study," Stroke, vol. 39, no. 11, pp. 2997-3002, 2008.

[57] S. Glagov, C. Zarins, D. P. Giddens, and D. N. Ku, "Hemodynamics and atherosclerosis. Insights and perspectives gained from studies of human arteries," Archives of Pathology and Laboratory Medicine, vol. 112, no. 10, pp. 1018-1031, 1988.

[58] D. N. Ku, D. P. Giddens, C. K. Zarins, and S. Glagov, "Pulsatile flow and atherosclerosis in the human carotid bifurcation. Positive correlation between plaque location and low and oscillating shear stress," Arteriosclerosis, Thrombosis, and Vascular Biology, vol. 5, no. 3, pp. 293-302, 1985.

[59] C. K. Zarins, D. P. Giddens, and B. K. Bharadvaj, "Carotid bifurcation atherosclerosis. Quantitative correlation of plaque localization with flow velocity profiles and wall shear stress," Circulation Research, vol. 53, no. 4, pp. 502-514, 1983.

[60] J. Frösen, A. Piippo, A. Paetau et al., "Remodeling of saccular cerebral artery aneurysm wall is associated with rupture: histological analysis of 24 unruptured and 42 ruptured cases," Stroke, vol. 35, no. 10, pp. 2287-2293, 2004.

[61] A. M. Malek, S. L. Alper, and S. Izumo, "Hemodynamic shear stress and its role in atherosclerosis," Journal of the American Medical Association, vol. 282, no. 21, pp. 2035-2042, 1999.

[62] D. Kaiser, M. A. Freyberg, and P. Friedl, "Lack of hemodynamic forces triggers apoptosis in vascular endothelial cells," Biochemical and Biophysical Research Communications, vol. 231, no. 3, pp. 586-590, 1997.

[63] A. A. Valencia, A. M. Guzmán, E. A. Finol, and C. H. Amon, "Blood flow dynamics in saccular aneurysm models of the basilar artery," Journal of Biomechanical Engineering, vol. 128, no. 4, pp. 516-526, 2006.

[64] A. Kamiya and T. Togawa, "Adaptive regulation of wall shear stress to flow change in the canine carotid artery," The American Journal of Physiology, vol. 239, no. 1, pp. H14-H21, 1980.

[65] P. C. Smiesko and V. Johnson, "The arterial lumen is controlled by flow-related shear stress," Physiology, vol. 8, pp. 3438, 1993.

[66] F. Tronc, M. Wassef, B. Esposito, D. Henrion, S. Glagov, and A. Tedgui, "Role of NO in flow-induced remodeling of the rabbit common carotid artery," Arteriosclerosis, Thrombosis, and Vascular Biology, vol. 16, no. 10, pp. 1256-1262, 1996.

[67] C. K. Zarins, M. A. Zatina, and D. P. Giddens, "Shear stress regulation of artery lumen diameter in experimental atherogenesis," Journal of Vascular Surgery, vol. 5, no. 3, pp. 413-420, 1987.

[68] J. R. Cebral, F. Mut, J. Weir, and C. M. Putman, "Association of hemodynamic characteristics and cerebral aneurysm rupture," American Journal of Neuroradiology, vol. 32, no. 2, pp. 264-270, 2011.

[69] G. N. Foutrakis, H. Yonas, and R. J. Sclabassi, "Saccular aneurysm formation in curved and bifurcating arteries," American Journal of Neuroradiology, vol. 20, no. 7, pp. 13091317, 1999.

[70] J. R. Cebral, M. Hernandez, and A. F. Frangi, “Computational analysis of blood flow dynamics in cerebral aneurysms from CTA and 3D rotational angiography image data," in Proceedings of the International Congress on Computational Bioengineering, pp. 191-198, Zaragoza, Spain, 2003.

[71] A. Valencia, H. Morales, R. Rivera, E. Bravo, and M. Galvez, "Blood flow dynamics in patient-specific cerebral aneurysm models: the relationship between wall shear stress and aneurysm area index," Medical Engineering and Physics, vol. 30, no. 3, pp. 329-340, 2008.
[72] G. Lu, L. Huang, X. L. Zhang et al., "Influence of hemodynamic factors on rupture of intracranial aneurysms: patientspecific 3D mirror aneurysms model computational fluid dynamics simulation," American Journal of Neuroradiology, vol. 32, no. 7, pp. 1255-1261, 2011.

[73] R. Torii, M. Oshima, T. Kobayashi, K. Takagi, and T. E. Tezduyar, "Fluid-structure interaction modeling of aneurysmal conditions with high and normal blood pressures," Computational Mechanics, vol. 38, no. 4-5, pp. 482-490, 2006.

[74] R. Torii, M. Oshima, T. Kobayashi, K. Takagi, and T. E. Tezduyar, "Influencing factors in image-based fluid-structure interaction computation of cerebral aneurysms," International Journal for Numerical Methods in Fluids, vol. 65, no. 1-3, pp. 324-340, 2011.

[75] J. G. Isaksen, Y. Bazilevs, T. Kvamsdal et al., "Determination of wall tension in cerebral artery aneurysms by numerical simulation," Stroke, vol. 39, no. 12, pp. 3172-3178, 2008.

[76] C. Groden, J. Laudan, S. Gatchell, and H. Zeumer, "Threedimensional pulsatile flow simulation before and after endovascular coil embolization of a terminal cerebral aneurysm," Journal of Cerebral Blood Flow and Metabolism, vol. 21, no. 12, pp. 1464-1471, 2001.

[77] S. I. Stiver, P. J. Porter, R. A. Willinsky, and M. C. Wallace, "Acute human histopathology of an intracranial aneurysm treated using Guglielmi detachable coils: case report and review of the literature," Neurosurgery, vol. 43, no. 5, pp. 1203-1207, 1998.

[78] H. Tenjin, S. Fushiki, Y. Nakahara et al., "Effect of Guglielmi detachable coils on experimental carotid artery aneurysms in primates," Stroke, vol. 26, no. 11, pp. 2075-2080, 1995.

[79] M. B. Horowitz, P. D. Purdy, D. Burns, and D. Bellotto, "Scanning electron microscopic findings in a basilar tip aneurysm embolized with guglielmi detachable coils," American Journal of Neuroradiology, vol. 18, no. 4, pp. 688-690, 1997.

[80] S. Shimizu, A. Kurata, M. Takano et al., "Tissue response of a small saccular aneurysm after incomplete occlusion with a Guglielmi detachable coil," American Journal of Neuroradiology, vol. 20, no. 4, pp. 546-548, 1999.

[81] H. S. Byun and K. Rhee, "CFD modeling of blood flow following coil embolization of aneurysms," Medical Engineering and Physics, vol. 26, no. 9, pp. 755-761, 2004.

[82] S. Ahmed, I. D. Šutalo, and H. Kavnoudias, "Hemodynamics and stress distribution in a cerebral aneurysm partially blocked with coils," in Proceedings of the 5th International Conference on CFD in the Process Industries CSIRO, pp. 1315, Melbourne, Australia, 2006.

[83] N. M. P. Kakalis, A. P. Mitsos, J. V. Byrne, and Y. Ventikos, "The haemodynamics of endovascular aneurysm treatment: a computational modelling approach for estimating the influence of multiple coil deployment," IEEE Transactions on Medical Imaging, vol. 27, no. 6, Article ID 4531637, pp. 814824, 2008.

[84] A. P. Mitsos, N. M. P. Kakalis, Y. P. Ventikos, and J. V. Byrne, "Haemodynamic simulation of aneurysm coiling in an anatomically accurate computational fluid dynamics model: technical note," Neuroradiology, vol. 50, no. 4, pp. 341-347, 2008.

[85] A. Narracott, S. Smith, P. Lawford et al., "Development and validation of models for the investigation of blood clotting in idealized stenoses and cerebral aneurysms," Journal of Artificial Organs, vol. 8, no. 1, pp. 56-62, 2005.

[86] Y. Wei, S. Cotin, J. Allard, L. Fang, C. Pan, and S. Ma, "Interactive blood-coil simulation in real-time during aneurysm 
embolization," Computers and Graphics, vol. 35, no. 2, pp. 422-430, 2011.

[87] K. Baráth, F. Cassot, D. A. Rüfenacht, and J. H. D. Fasel, "Anatomically shaped internal carotid artery aneurysm in vitro model for flow analysis to evaluate stent effect," American Journal of Neuroradiology, vol. 25, no. 10, pp. 17501759, 2004.

[88] K. Baráth, F. Cassot, J. H. D. Fasel, M. Ohta, and D. A. Rüfenacht, "Influence of stent properties on the alteration of cerebral intra-aneurysmal haemodynamics: flow quantification in elastic sidewall aneurysm models," Neurological Research, vol. 27, no. 1, pp. S120-S128, 2005.

[89] B. B. Lieber, A. P. Stancampiano, and A. K. Wakhloo, "Alteration of hemodynamics in aneurysm models by stenting: influence of stent porosity," Annals of Biomedical Engineering, vol. 25, no. 3, pp. 460-469, 1997.

[90] K. Rhee, M. H. Han, and S. H. Cha, "Changes of flow characteristics by stenting in aneurysm models: influence of aneurysm geometry and stent porosity," Annals of Biomedical Engineering, vol. 30, no. 7, pp. 894-904, 2002.

[91] B. B. Lieber, V. Livescu, L. N. Hopkins, and A. K. Wakhloo, "Particle image velocimetry assessment of stent design influence on intra-aneurysmal flow," Annals of Biomedical Engineering, vol. 30, no. 6, pp. 768-777, 2002.

[92] S. C. M. Yu and J. B. Zhao, "A steady flow analysis on the stented and non-stented sidewall aneurysm models," Medical Engineering and Physics, vol. 21, no. 3, pp. 133-141, 1999.

[93] T. M. Liou, W. C. Chang, and C. C. Liao, "LDV measurements in lateral model aneurysms of various sizes," Experiments in Fluids, vol. 23, no. 4, pp. 317-324, 1997.

[94] S. Tateshima, Y. Murayama, J. P. Villablanca et al., "In vitro measurement of fluid-induced wall shear stress in unruptured cerebral aneurysms harboring blebs," Stroke, vol. 34, no. 1, pp. 187-192, 2003.

[95] M. Aenis, A. P. Stancampiano, A. K. Wakhloo, and B. B. Lieber, "Modeling of flow in a straight stented and nonstented side wall aneurysm model," Journal of Biomechanical Engineering, vol. 119, no. 2, pp. 206-212, 1997.

[96] M. Ohta, S. G. Wetzel, P. Dantan et al., "Rheological changes after stenting of a cerebral aneurysm: a finite element modeling approach," CardioVascular and Interventional Radiology, vol. 28, no. 6, pp. 768-772, 2005.

[97] J. R. Cebral, M. Hernandez, A. F. Frangi, C. M. Putman, R. Pergolizzi, and J. E. Burgess, "Subject-specific modeling of intracranial aneurysms," in Medical Imaging, Proceedings of SPIE, pp. 319-327, 2004.

[98] J. R. Cebral and R. Loehner, "Flow visualization on unstructured grids using geometrical cuts, vortex detection and shock surfaces," in Proceedings of the 39th AIAA Aerospace Sciences Meeting and Exhibit, Reno, Nev, USA, 2001.

[99] J. R. Cebral and R. Lhner, "From medical images to anatomically accurate finite element grids," International Journal for Numerical Methods in Engineering, vol. 51, no. 8, pp. 9851008, 2001.

[100] L. D. Jou, C. M. Quick, W. L. Young et al., "Computational approach to quantifying hemodynamic forces in giant cerebral aneurysms," American Journal of Neuroradiology, vol. 24, no. 9, pp. 1804-1810, 2003.

[101] R. Löhner, "Regridding surface triangulations," Journal of Computational Physics, vol. 126, no. 1, pp. 1-10, 1996.

[102] R. Lohner, "Automatic unstructured grid generators," Finite Elements in Analysis and Design, vol. 25, no. 1-2, pp. 111-134, 1997.
[103] J. R. Cebral, F. Mut, M. Raschi et al., "Aneurysm rupture following treatment with flow-diverting stents: computational hemodynamics analysis of treatment," American Journal of Neuroradiology, vol. 32, pp. 27-33, 2011.

[104] S. Appanaboyina, F. Mut, R. Löhner, C. Putman, and J. Cebral, "Simulation of intracranial aneurysm stenting: techniques and challenges," Computer Methods in Applied Mechanics and Engineering, vol. 198, no. 45-46, pp. 35673582, 2009.

[105] S. Appanaboyina, F. Mut, R. Löhner, C. M. Putman, and J. R. Cebral, "Computational fluid dynamics of stented intracranial aneurysms using adaptive embedded unstructured grids," International Journal for Numerical Methods in Fluids, vol. 57, no. 5, pp. 475-493, 2008.

[106] J. R. Cebral and R. Löhner, "Efficient simulation of blood flow past complex endovascular devices using an adaptive embedding technique," IEEE Transactions on Medical Imaging, vol. 24, no. 4, pp. 468-476, 2005.

[107] L. Augsburger, P. Reymond, D. A. Rufenacht, and N. Stergiopulos, "Intracranial stents being modeled as a porous medium: flow simulation in stented cerebral aneurysms," Annals of Biomedical Engineering, vol. 39, pp. 850-863, 2011.

[108] B. Chopard, R. Ouared, D. A. Ruefenacht, and H. Yilmaz, "Lattice Boltzmann modeling of thrombosis in giant aneurysms," International Journal of Modern Physics C, vol. 18, no. 4, pp. 712-721, 2007.

[109] B. Chopard, R. Ouared, and D. A. Rüfenacht, "A lattice Boltzmann simulation of clotting in stented aneursysms and comparison with velocity or shear rate reductions," Mathematics and Computers in Simulation, vol. 72, no. 2-6, pp. 108-112, 2006.

[110] R. Ouared, B. Chopard, B. Stahl, D. A. Rüfenacht, H. Yilmaz, and G. Courbebaisse, "Thrombosis modeling in intracranial aneurysms: a lattice Boltzmann numerical algorithm," Computer Physics Communications, vol. 179, no. 1-3, pp. 128-131, 2008.

[111] S. Tateshima, J. Grinstead, S. Sinha et al., "Intraaneurysmal flow visualization by using phase-contrast magnetic resonance imaging: feasibility study based on a geometrically realistic in vitro aneurysm model," Journal of Neurosurgery, vol. 100, no. 6, pp. 1041-1048, 2004.

[112] Y. P. Gobin, J. L. Counord, P. Flaud, and J. Duffaux, "In vitro study of haemodynamics in a giant saccular aneurysm model: influence of flow dynamics In the parent vessel and effects of coil embolisation," Neuroradiology, vol. 36, no. 7, pp. 530536, 1994.

[113] T. M. Liou and S. N. Liou, "A review on in vitro studies of hemodynamic characteristics in terminal and lateral aneurysm models," Proceedings of the National Science Council, Republic of China. Part B, Life sciences, vol. 23, no. 4, pp. 133$148,1999$.

[114] T. Satoh, K. Onoda, and S. Tsuchimoto, "Visualization of intraaneurysmal flow patterns with transluminal flow images of 3D MR angiograms in conjunction with aneurysmal configurations," American Journal of Neuroradiology, vol. 24, no. 7, pp. 1436-1445, 2003.

[115] H. Ujiie, H. Tachibana, O. Hiramatsu et al., "Effects of size and shape (aspect ratio) on the hemodynamics of saccular aneurysms: a possible index for surgical treatment of intracranial aneurysms," Neurosurgery, vol. 45, no. 1, pp. 119-130, 1999.

[116] A. J. Ringer, D. K. Lopes, A. S. Boulos, L. R. Guterman, and L. N. Hopkins, "Current techniques for endovascular treatment 
of intracranial aneurysms," Seminars in Cerebrovascular Diseases and Stroke, vol. 1, pp. 39-51, 2001.

[117] S. H. Kim, M. H. Han, I. K. Yu, S. H. Lee, and K. H. Chang, "Experimental model for creation of carotid artery aneurysms in dogs," Journal of the Korean Radiological Society, vol. 35, pp. 703-707, 1996.

[118] K. Perktold, R. Peter, and M. Resch, "Pulsatile non-Newtonian blood flow simulation through a bifurcation with an aneurysm," Biorheology, vol. 26, no. 6, pp. 1011-1030, 1989.

[119] K. Perktold and G. Rappitsch, "Computer simulation of local blood flow and vessel mechanics in a compliant carotid artery bifurcation model," Journal of Biomechanics, vol. 28, no. 7, pp. 845-856, 1995.

[120] D. A. Steinman, "Image-based computational fluid dynamics modeling in realistic arterial geometries," Annals of Biomedical Engineering, vol. 30, no. 4, pp. 483-497, 2002.

[121] G. R. Stuhne and D. A. Steinman, "Finite-element modeling of the hemodynamics of stented aneurysms," Journal of Biomechanical Engineering, vol. 126, no. 3, pp. 382-387, 2004.

[122] L. Dempere-Marco, E. Oubel, M. Castro, C. Putman, A. Frangi, and J. Cebral, "CFD analysis incorporating the influence of wall motion: application to intracranial aneurysms," Lecture Notes in Computer Science, vol. 4191, pp. 438-445, 2006.

[123] E. Oubel, M. De Craene, C. M. Putman, J. R. Cebral, and A. F. Frangi, "Analysis of intracranial aneurysm wall motion and its effects on hemodynamic patterns," in Medical Imaging, vol. 6511 of Proceedings of SPIE, p. 65112A, San Diego, Calif, USA, February 2007.

[124] K. Takizawa, C. Moorman, S. Wright et al., "Patient-specific arterial fluid-structure interaction modeling of cerebral aneurysms," International Journal for Numerical Methods in Fluids, vol. 65, no. 1-3, pp. 308-323, 2011.

[125] C. A. Figueroa, S. Baek, I. E. Vignon-Clementel, J. D. Humphrey, and C. A. Taylor, "Towards patient-specific modeling I: hemodynamics in a growing aneurysm," in Medical Imaging, vol. 6143 of Proceedings of SPIE, p. 61430K, San Diego, Calif, USA, 2006.

[126] R. L. Gleason and J. D. Humphrey, "A mixture model of arterial growth and remodeling in hypertension: altered muscle tone and tissue turnover," Journal of Vascular Research, vol. 41, no. 4, pp. 352-363, 2004.

[127] R. L. Gleason and J. D. Humphrey, "Effects of a sustained extension on arterial growth and remodeling: a theoretical study," Journal of Biomechanics, vol. 38, no. 6, pp. 1255-1261, 2005.

[128] S. Baek, C. A. Figueroa, C. A. Taylor, and J. D. Humphrey, "Towards patient-specific modeling II. Biomechanics of a growing aneurysm," in Medical Imaging, vol. 6143 of Proceedings of SPIE, p. 61432C, San Diego, Calif, USA, 2006.

[129] R. A. Peattie, T. J. Riehle, and E. I. Bluth, "Pulsatile flow in fusiform models of abdominal aortic aneurysms: flow fields, velocity patterns and flow-induced wall stresses," Journal of Biomechanical Engineering, vol. 126, no. 4, pp. 438-446, 2004.

[130] S. Prakash and C. R. Ethier, "Requirements for mesh resolution in 3D computational hemodynamics," Journal of Biomechanical Engineering, vol. 123, no. 2, pp. 134-144, 2001.

[131] A. V. Salsac, S. R. Sparks, and J. C. Lasheras, "Hemodynamic changes occurring during the progressive enlargement of abdominal aortic aneurysms," Annals of Vascular Surgery, vol. 18 , no. 1, pp. 14-21, 2004. 


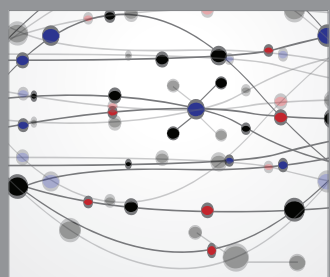

The Scientific World Journal
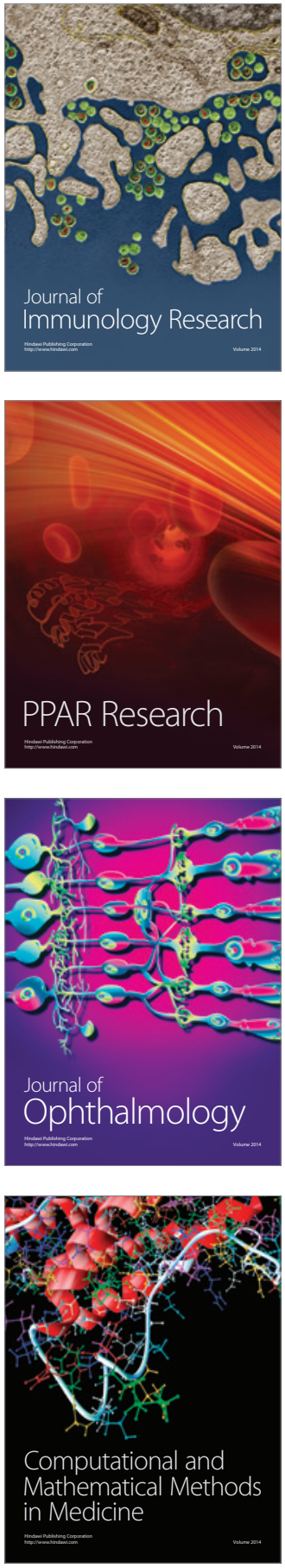

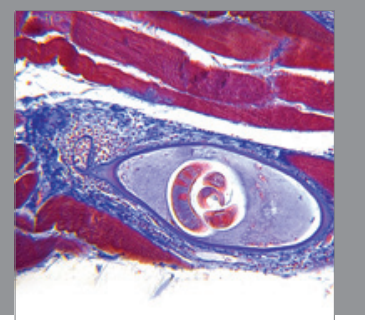

Gastroenterology

Research and Practice
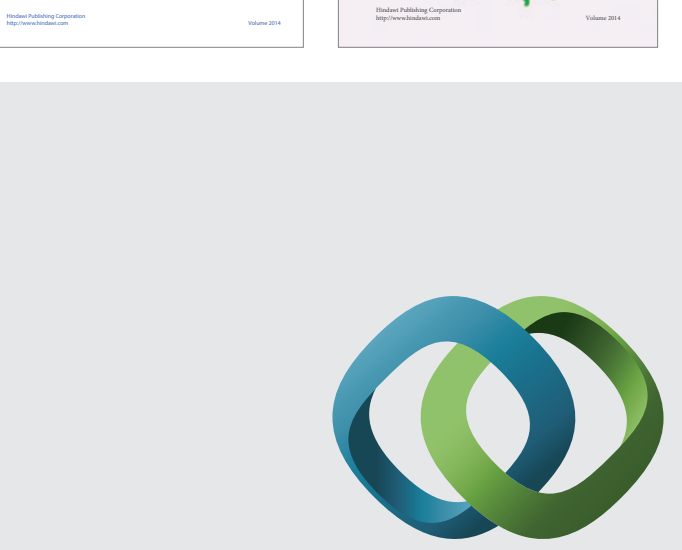

\section{Hindawi}

Submit your manuscripts at

http://www.hindawi.com
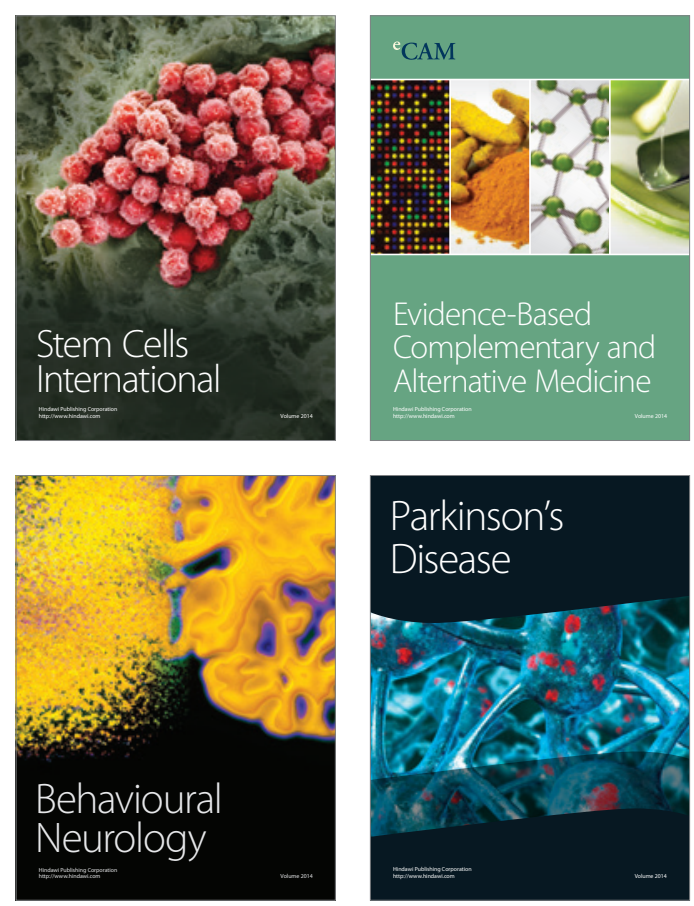

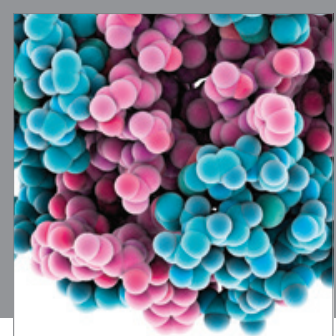

Journal of
Diabetes Research

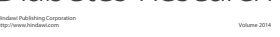

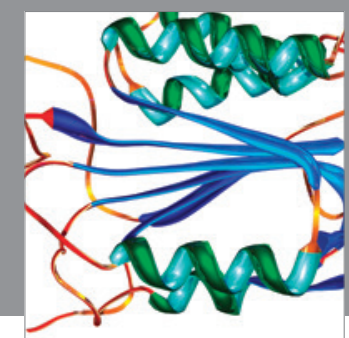

Disease Markers
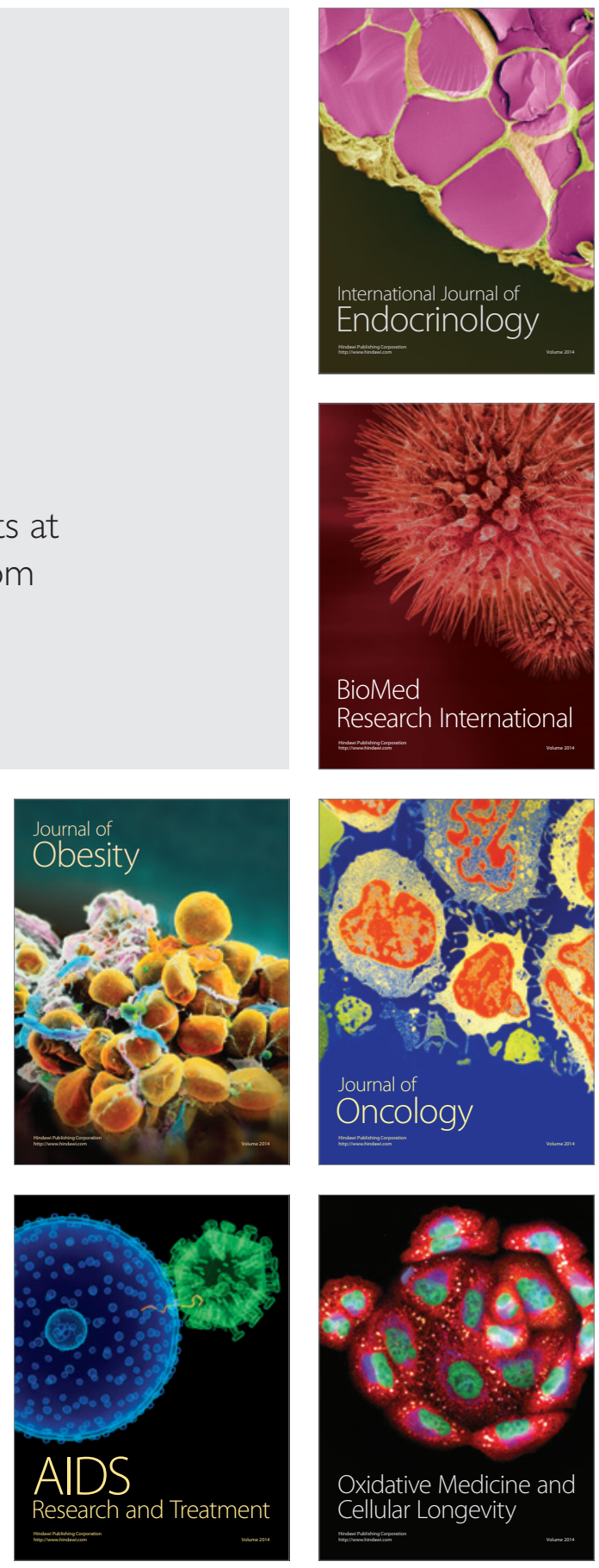